\title{
Development of a quantification method for fouling deposits using phosphorescence
}

\author{
H. Deponte ${ }^{1}\left(\mathbb{D} \cdot\right.$ W. Augustin ${ }^{1} \cdot$ S. Scholl ${ }^{1}$
}

Received: 9 March 2020 / Accepted: 11 March 2021 / Published online: 26 March 2021

(C) The Author(s) 2021

\begin{abstract}
Particulate fouling on structured surfaces is typically quantified using the integral thermal or mass-based fouling resistance. The observed geometries may be structures that can improve the heat transfer in heat exchangers (e.g., dimples), cavities in components, or more complex geometries. However, due to limited accessibility or the requirement for a locally resolved measurement, the existing quantification methods may not be applicable to structured surfaces. For this reason, a new method is needed for the quantification for fouling deposits. In this study, dimpled surfaces were evaluated by measuring the integral thermal and mass-based fouling resistance and comparing it with the local fouling resistance inside and around the dimple. This comparison was carried out online with the Phosphorescent Fouling Quantification method developed for this purpose, using phosphorescent particles to quantify the deposited mass. The mass-based fouling resistance can be calculated using computer-aided image analysis. The measurements for the evaluation were conducted on dimpled surfaces, which produced a characteristic fouling pattern. With the new method a reduced surface coverage from up to $33.3 \%$ was observed, which led to lower fouling resistances downstream of the dimple compared to a plain surface. These results confirm earlier numerical and experimental findings, suggesting an advantage of dimpled surfaces over other surface structures with respect to thermo-hydraulic efficiency as well as reduced fouling. Thus, the Phosphorescent Fouling Quantification method provides the possibility of calculating values for local fouling resistances on structured surfaces, as well as the possibility of optimizing surface structures to minimize fouling propensity.
\end{abstract}

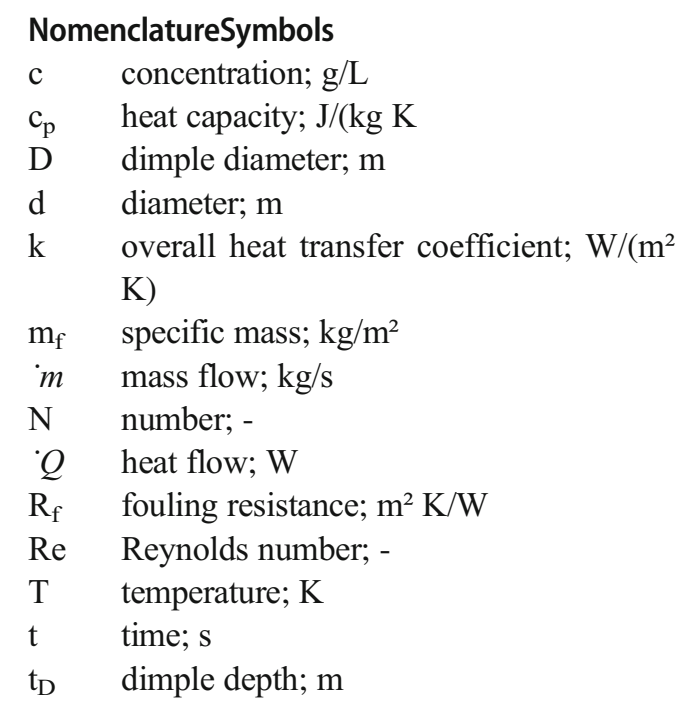

H. Deponte h.deponte@tu-braunschweig.de

1 Institute for Chemical and Thermal Process Engineering (ICTV), Technische Universität Braunschweig, Langer Kamp 7, 38106 Braunschweig, Germany

$\begin{array}{ll}\text { u } & \text { fluid velocity; } \mathrm{m} / \mathrm{s} \\ \mathrm{V} & \text { volume; } \mathrm{m}^{3} \\ \mathrm{X} & \text { layer thickness; } \mathrm{m} \\ \Delta & \text { difference; - } \\ \varepsilon & \text { void fraction; - } \\ \lambda & \text { thermal conductivity; } \mathrm{W} /(\mathrm{m} \mathrm{K}) \\ \rho & \text { density; } \mathrm{kg} / \mathrm{m}^{3} \\ \psi & \text { sphericity; - } \\ \text { Subscripts } \\ 0 & \text { without fouling } \\ \text { cell } & \text { observed cell volume } \\ \mathrm{f} & \text { fouling material, with fouling } \\ \mathrm{h} & \text { hydraulic } \\ \mathrm{m} & \text { mass based, logarithmic mean } \\ \mathrm{p} & \text { particle } \\ \mathrm{p}, 50 & \text { mean particle value } \\ \text { pfq } & \text { based on PFQ method } \\ \text { Susp. } & \text { suspension } \\ \text { th } & \text { thermal } \\ \text { tot } & \text { overall fouling layer } \\ \text { w } & \text { water }\end{array}$

Abbreviations

LPD Local Phosphorescence Detection 
PFQ Phosphorescent Fouling Quantification

$\mathrm{S} 26 \mathrm{~B}$ Test plate with one dimple, $\mathrm{t}_{\mathrm{D}} / \mathrm{D}=0.26$

\section{Introduction}

\subsection{Particulate fouling on structured surfaces}

Particulate fouling on heat exchanger surfaces is one of the main causes of efficiency problems, so its prevention is a major challenge in the processing industry [1]. The decrease in heat transfer due to fouling often leads to overdesign and higher energy and maintenance costs [2]. Therefore, evaluation of the long-term efficiency of heat exchangers must take into account the fouling propensity in addition to the thermohydraulic efficiency, which is based on the ratio of heat transfer to flow resistance. Heat exchangers with dimpled surfaces have a comparatively high thermo-hydraulic efficiency. They are used for cooling processes with river water, where the fouling probability is high due to suspended particles (e.g., sand or corrosion products) or in processes where particleladen fluids must be cooled or heated [3].

The mechanisms of particulate fouling on plate heat exchangers have been described by Grandgeorge et al. [4], among others. Diffusion or forces of inertia transport suspended particles from the bulk fluid to the wall surface, where the particles subsequently adhere mainly by van der Waals and electrostatic forces [5]. A fouling layer forms when the particle deposition rate is higher than the removal rate. Technically, the opposite case of a high removal rate can be used to clean a soiled surface [6]. This higher removal can be achieved by increasing the shear stresses through increases in the fluid velocity and the use of surface structuring. Investigation of the effect of structuring on the various fouling mechanisms (i.e., deposition, suppression, or removal) requires quantification of the fouling layers on a local basis.

\subsection{Quantification of particulate fouling}

Most studies use the thermal fouling resistance for the quantification of particulate fouling on heat exchanging surfaces (e.g. [7]). The fouling resistance is measured either integrally or locally with the corresponding temperatures, but the accuracy of the method in both cases is highly dependent on the measurement technology. Furthermore, a heat transferring surface is always necessary, since the heat flux is required for the calculation of the overall heat transfer coefficient and, therefore, for calculation of the thermal fouling resistance.

Particle fouling can also occur without the presence of a temperature gradient, for example, in piping systems without accompanied heat transfer. For isothermal processes with the formation of fouling layers, the mass-based fouling resistance is applicable. Besides the material parameters, the mass of the fouling layer has to be determined. This method is invasive in most cases, and it destroys the fouling layer due to drying or extraction of the layer. The fouling process also has to be interrupted for analysis. Overall, the result is highly influenced by the measurement procedure.

\subsection{Thermal fouling resistance}

The integral thermal fouling resistance is based on temperature measurements of the inlet and outlet streams of the product and heating fluid in the flow channel. The overall heat transfer coefficient for the clean channel $\mathrm{k}_{0}$ is first calculated from the heat flow $Q$ between the two streams, the heat transfer area $\mathrm{A}$ and the logarithmic mean temperature difference $\Delta \mathrm{T}_{\mathrm{m}}$ of the integrally measured temperatures:

$k_{0}=\frac{Q}{A \Delta T_{m}}$

The heat flow through the wall $Q$ is calculated either from the heat emitted by the heating fluid or the heat absorbed by the product stream. The temperature difference $\Delta \mathrm{T}_{\mathrm{i}}$ in Eq. (2) is determined between the inlet and outlet flow of the channel. The temperature-dependent data for the density $\rho$, which are necessary for calculation of the mass flow $m_{i}$ and the heat capacity $c_{p}$, are taken from the literature according to the arithmetic mean temperature of the fluid. One assumption is that the suspended particles, at the concentration of $0.2 \mathrm{~g} / \mathrm{L}$ used, do not affect the properties of the water. The calculated values of the heat flows of the product and heating side should be identical if no heat loss occurs.

$Q_{i}=m_{i} c_{p, i} \Delta T_{i}$

During a fouling experiment, the time-dependent overall heat transfer coefficient is also determined. This is now influenced by the fouling process and defines the heat transfer in the heat exchanger, including the influence of the deposited fouling layer. The value of the new overall heat transfer coefficient $\mathrm{k}_{\mathrm{f}}$ is smaller than $\mathrm{k}_{0}$ due to the additional thermal resistance of the fouling layer, the so-called thermal fouling $R_{f, t h}=\frac{1}{k_{f}}-\frac{1}{k_{0}}$

\subsection{Mass-based fouling resistance}

The parameters for the mass-based fouling resistance $R_{f, m}$ are achieved with an invasive method described by Deponte et al. [8]. The specific mass $m_{f}$ of the deposited particles is determined by extracting the dried fouling layer from the plate with adhesive tape (A tape with the geometry of $18 \mathrm{~mm} \times 18 \mathrm{~mm}$ is 
used). Two tapes are placed on the surface of the test plate upstream and downstream of the dimple. The particles adhering to the adhesive tape are weighed with an accuracy of $0.1 \mathrm{mg}$, which equals an accuracy of the specific mass $\mathrm{m}_{\mathrm{f}}$ of $0.03 \mathrm{~g} / \mathrm{m}^{2}$.

The equation for the mass-based fouling resistance includes the thermal conductivity $\lambda_{\mathrm{f} \text { tot }}$ and the thickness $\mathrm{x}_{\mathrm{f}}$ of the overall fouling layer [9]:

$R_{f, m}=\frac{x_{f}}{\lambda_{f, t o t}}$

The overall thermal conductivity $\lambda_{\mathrm{f}, \mathrm{tot}}$ is defined by the thermal conductivity of the fouling material $\lambda_{\mathrm{f}}$ and the thermal conductivity of the fluid (in this case, water $\lambda_{\mathrm{w}}$ ) that fills the cavities between the particles and is part of the stagnant layer [10]. The proportion of the involved thermal conductivities results from the void fraction $\varepsilon$, which defines the free volume inside the deposit. The void fraction is calculated by observing a defined volume of the fouling layer, the cell volume $V_{\text {cell }}$. The occupied volume is calculated using the number $\mathrm{N}_{\mathrm{p}}$ and volume $\mathrm{V}_{\mathrm{p}}$ of the particles in the observed cell The occupied volume of a cell is defined in Eq. (5).

$(1-\varepsilon)=\frac{N_{p} V_{p}}{V_{\text {cell }}}$

The value for the packing factor $(1-\varepsilon)$ has to be assumed in order to calculate the mass-based fouling resistance. Due to their sphericity, the particles will arrange in sphere-like packings. This limits the possible values for the packing factor to the range of $(1-\varepsilon)=0.52$ (simple cubic) to $(1-\varepsilon)=0.74$ (face-centered cubic). A random packing generally has a packing factor of around $(1-\varepsilon)=0.64$ [11]. The further evaluations of fouling resistances in this study used this value for the calculation of $\mathrm{R}_{\mathrm{f}, \mathrm{pfq}}$ and $\mathrm{R}_{\mathrm{f}, \mathrm{m}}$.

In the proportion given by the void fraction of a random packing, considered as homogeneous, the thermal conductivities are described mathematically as series-connected resistances to calculate the overall thermal conductivity $\lambda_{\mathrm{f}, \text { tot }}[12]$.

$\frac{1}{\lambda_{f, t o t}}=\frac{1-\varepsilon}{\lambda_{f}}+\frac{\varepsilon}{\lambda_{w}}$

Thus, the mass-based fouling resistance related to the height of the fouling layer follows from insertion of Eq. (6) into Eq. (4).

$R_{f, m}=x_{f} *\left(\frac{1-\varepsilon}{\lambda_{\mathrm{f}}}+\frac{\varepsilon}{\lambda_{\mathrm{w}}}\right)$

The mean height $\mathrm{x}_{\mathrm{f}}$ of the fouling layer, necessary for the determination of the mass-based fouling resistance, is calculated with the specific mass $\mathrm{m}_{\mathrm{f}}$ and density of the deposited particles $\rho_{\mathrm{f}}$. The value is corrected with the void fraction $\varepsilon$. $x_{f}=\frac{m_{f}}{\rho_{f}(1-\varepsilon)}$

The mass-based fouling resistance then follows by combining Eq. (8) and Eq. (7).

$R_{f, m}=\frac{m_{f}}{\rho_{f}(1-\varepsilon)} *\left(\frac{1-\varepsilon}{\lambda_{\mathrm{f}}}+\frac{\varepsilon}{\lambda_{\mathrm{w}}}\right)$

This method is invasive and destroys the fouling layer, so it is the last applied method. This analytical method therefore differs from the others because it can only be used after completion of the experiment. By contrast, the PFQ method and the method for the thermal fouling resistance allow the possibility of obtaining results online during the experiment.

\section{Methods}

\subsection{Phosphorescent fouling quantification (PFQ) method}

This study introduces a new method for the evaluation of heat transfer surfaces, the Phosphorescent Fouling Quantification (PFQ) method. This method uses the luminescence of particles which is also used in several optical imaging techniques in microfluidics [13], e.g., with luminescent crystalline zinc sulfide as a tracer to assess the cleaning results of jet cleaning processes [14] or to detect proteinaceous fouling layers by using their excitability with UV-light [15]. The PFQ method quantifies the deposited mass by the use of phosphorescent particles as the fouling material. This is an optical non-invasive method for the online determination of local mass-based fouling resistances.

The principle of the PFQ method is based in the Local Phosphorescence Detection (LPD) method first introduced by Schöler et al. [16]. The LPD was used for the evaluation of the local cleaning efficiency of pulsed-flow cleaning procedures for Cleaning in Place (CIP) processes in the food industry. Phosphorescent crystalline zinc sulfide was integrated into the applied fouling layer as a tracer. The cleaning progression was monitored optically by detection of the emitted light of the tracer and was assessed by the resulting coverage of the surface. This method was evaluated against other methods for studying CIP mechanisms by Gordon et al. [17] and was deemed suitable for measuring the cleaning time.

The PFQ method uses phosphorescent particles that are dispersed in an aqueous suspension at a concentration of $\mathrm{c}=$ $0.2 \mathrm{~g} / \mathrm{L}$. These tracer particles function simultaneously as the fouling material. The particles are copper-doped zinc sulfide crystals (Lumilux green SN-F5, Honeywell). Figure 1 shows an image of the particles taken with the scanning electron microscope. These particles are used as they are a widely used material for phosphorescent tracer particles and thus available 




Fig. 1 Image of the Lumilux green SN-F5 particles taken with a scanning electron microscope

in many specifications at a reasonable price. Lumilux green SN-F5 exhibit a strong phosphorescence and provide a high sphericity of $\psi=0.88 \pm 0.07$. The sphericity indicates how spherical a solid is by comparing its surface area with that of a sphere of equal volume. The volume of the particles is determined with a scanning electron microscope by tilting the particle sample to create semi stereoscopic images.

The particles have a narrow particle size distribution, with a median diameter of $\mathrm{d}_{\mathrm{p}, 50}=1.14 \mu \mathrm{m}$ (Fig. 2). Determination of the mass-based fouling resistance requires a value for the density of the particles, which is $\rho_{\mathrm{f}}=4,090 \mathrm{~kg} / \mathrm{m}^{3}$. The copper activator produces a longduration green afterglow of the material after activation with visible light. This emitted light is detected with a camera encased in a suitable housing that excludes ambient light. The camera settings used in this study were: Aperture f/5.6, shutter speed 4" and ISO 1600. The images can be taken during the fouling process through the glass windows in the lid of the channel without interrupting or influencing the particulate flow and

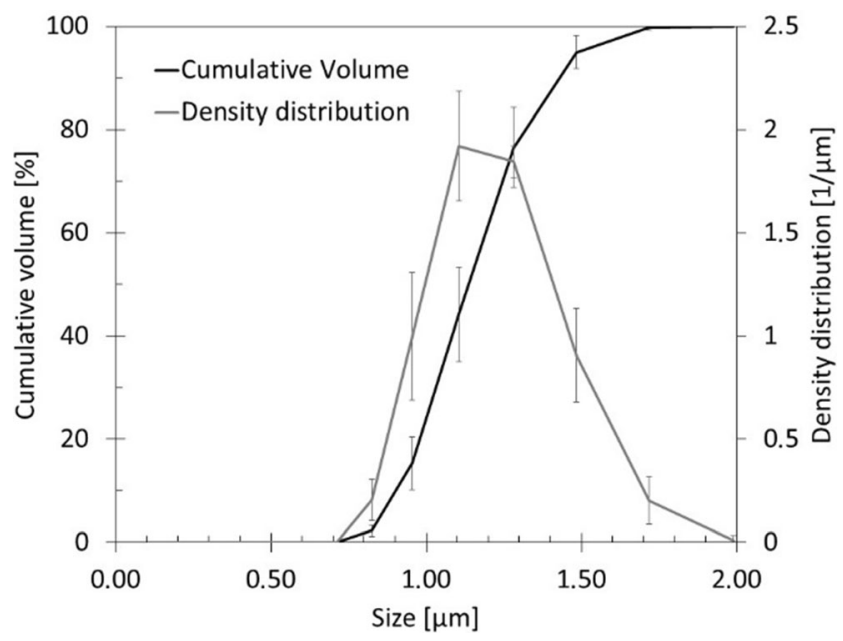

Fig. 2 Cumulative particle size distribution of Lumilux green SN-F5 deposition. Therefore, the fouling progression can be observed over time.

The captured images are processed using an analysis method created in Matlab ${ }^{\circledR}$ (Version R2016a 9.0.0.341360, MathWorks, 2016). This procedure is shown schematically in Fig. 3. The true color images are converted from the rgb color space into a grayscale intensity image by eliminating the information for hue and saturation. The luminance is retained, and its value for each pixel of the image is saved in a matrix. Therefore, the matrix has the same dimensions as the resolution of the image. The luminescence value is proportional to the number of particles deposited on the surface. The mathematical correlation of luminescence and the height of the fouling layer is deducted for each image from the calibration, with local height measurements performed with a digital microscope. Small particle layers, which formed during the investigations in this study, show a linear dependence between the layer height and the intensity of emitted light. The results are then displayed as a scaled heat map.

The use of the physical data of the fouling layer material allows conversion of the height of the fouling layer $\mathrm{x}_{\mathrm{f}}$ into the mass-based fouling resistance $\mathrm{R}_{\mathrm{f}, \mathrm{m}}$. To avoid confusion with other methods, the mass-based fouling resistance measured with the PFQ is indicated as $\mathrm{R}_{\mathrm{f}, \mathrm{pfq}}$.

$R_{f, p f q}=x_{f} *\left(\frac{1-\varepsilon}{\lambda_{\mathrm{f}}}+\frac{\varepsilon}{\lambda_{\mathrm{w}}}\right)$

The values for the temperature-dependent thermal conductivity of water are taken from [18]. The deposited particles have a thermal conductivity of $\lambda_{\mathrm{f}}=27.2 \mathrm{~W} /(\mathrm{m} \mathrm{K})$. The thermal conductivity of the solid material is also assumed to be temperature independent in the given temperature range.

The PFQ method is evaluated with experiments on particulate fouling on dimpled surfaces. The mass-based fouling resistance achieved with the PFQ is validated with the values of the mass-based fouling resistance, which are measured at the end of each experiment. The time-dependent fouling behavior, expressed during the course of the thermal fouling resistance, is compared with the online fouling resistances measured with the PFQ method.

\subsection{Experimental setup}

The experimental setup of the test rig used for the investigations of the fouling behavior of particles in aqueous suspensions on structured surfaces is described in detail by Deponte et al. [8]. Figure 4 shows the flow diagram of the particulate fouling plant.

The fouling experiments were conducted in a flow channel with a rectangular cross section of $4 \mathrm{~mm}$ height $\times 18 \mathrm{~mm}$ width, resulting in a hydraulic diameter of $d_{h}=6.55 \mathrm{~mm}$, 
Fig. 3 Image processing procedure of the Phosphorescent Fouling Quantification (PFQ) method

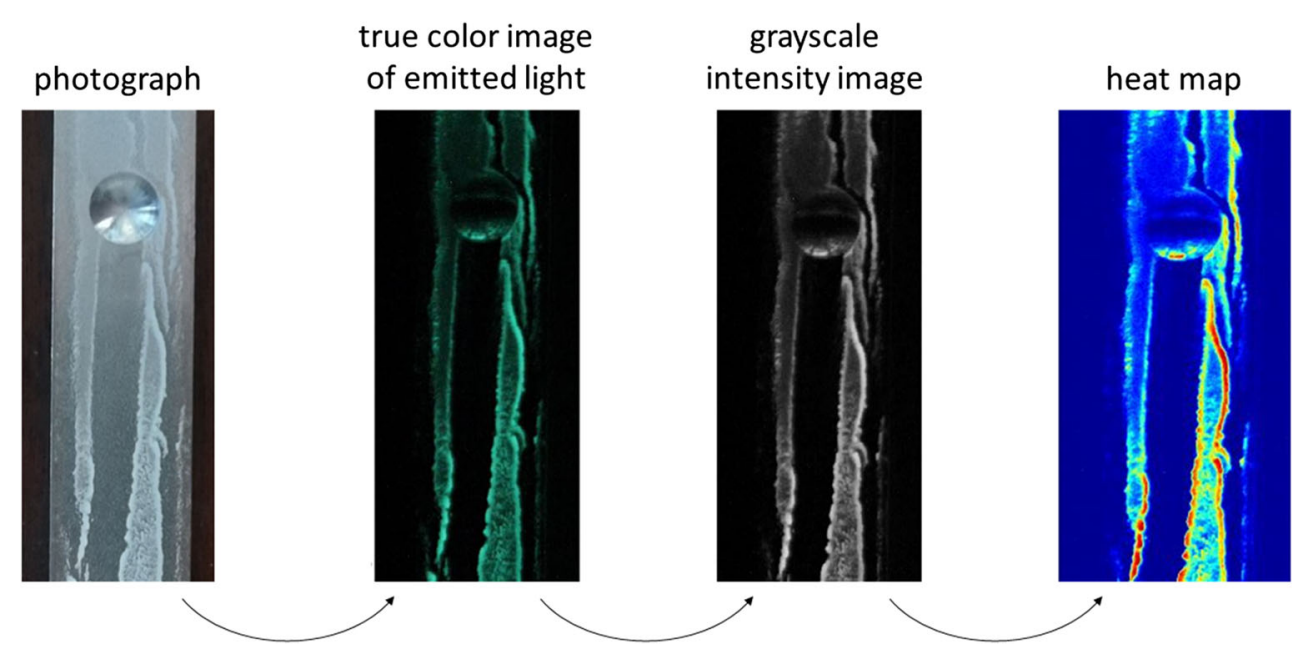

\subsection{Test plates}

Dimpled surfaces (see Fig. 7) were used to evaluate the PFQ method. The single dimpled is provided on a test plate, which is inserted in the measuring section of the flow channel.

Dimpled surfaces provide a local pattern for the fouling deposit due to the self-cleaning effect described by Kasper et al. [19]. Kasper et al. observed a trail downstream from the dimple, with a decreased particle deposit on the surface inside the trail in comparison to the area upstream of the dimple (see Fig. 8). The observations led to the hypothesis that

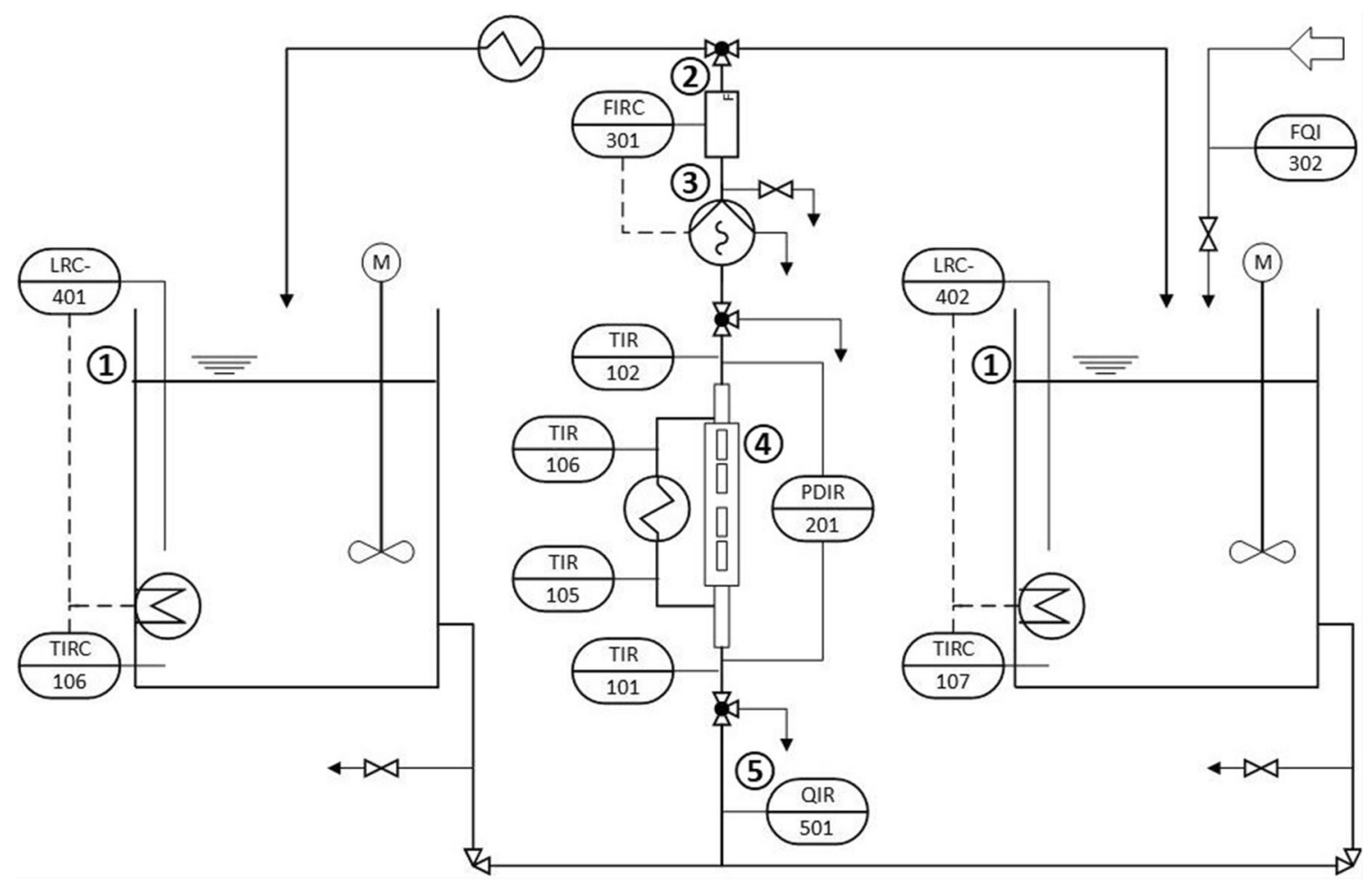

Fig. 4 Flow diagram of the particulate fouling test plant with (1) stirred and heated vessels, (2) magnetic flow meter, (3) progressive cavity pump, (4) flow channel and (5) turbidity sensor. (Adapted from [8]) 
Fig. 5 Image of the flow channel with the inlet, measuring section and outlet.(Adapted from [19])

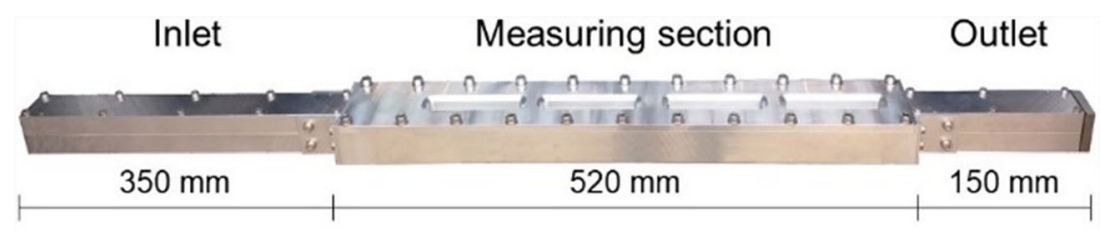

Flow direction $\rightarrow$ dimpled surfaces in heat exchangers are advantageous over other surface structures with respect to reduced fouling propensity.

The self-cleaning effect is numerically and experimentally visualized on the surface downstream of the dimple. The first quantification of the self-cleaning effect of dimples was shown by Deponte et al. [8], who measured the decrease in coverage of the surface.

In addition to the reduced fouling propensity, the thermohydraulic efficiency of dimpled surfaces is enhanced in comparison to a planar plate [20]. The thermo-hydraulic efficiency is defined as the ratio of heat transfer to flow resistance, which is used for the evaluation of structured surfaces in heat exchangers with and without dimples [21]. Dimples with a ratio of dimple depth $t_{\mathrm{D}}$ to diameter $\mathrm{D}$ of $\mathrm{t}_{\mathrm{D}} / \mathrm{D}=0.26$ were identified numerically as the best geometry with respect to thermohydraulic efficiency [22].

The test plates used for the experiments are $500 \mathrm{~mm}$ in length and fabricated from bright stainless steel (1.4301). The plate has one single dimple, with a ratio of dimple depth to diameter of $t_{D} / D=0.26$. The dimple is positioned on the plate $100 \mathrm{~mm}$ from the beginning, in the flow direction. It is designated in the following text as S26B.

A comprehensive approach for evaluating structured surfaces should consider the values for the fouling resistance. However, the methods for measuring the thermal or massbased fouling resistance are not applicable inside the cavity of the dimple. This is where the PFQ method is used.

\section{Results and discussion}

\subsection{Evaluation of the PFQ method}

The PFQ method is evaluated on dimpled surfaces. Spherical dimples are hypothesized to suppress the deposition of particles from a liquid stream or even to induce a permanent cleaning of the surface. In addition to the numerical studies

Table 1 Settings for the process parameters used for the experiments

\begin{tabular}{llrl}
\hline Parameter & Symbol & Setting & Unit \\
\hline Fluid velocity & $\mathrm{u}_{\text {Susp. }}$ & 1 & $\mathrm{~m} / \mathrm{s}$ \\
Reynolds number & $\mathrm{Re}_{\text {Susp. }}$ & 8,200 & - \\
Temperature & $\mathrm{T}_{\text {Susp. }}$ & 30 & ${ }^{\circ} \mathrm{C}$ \\
Time & $\mathrm{t}$ & 1 & $\mathrm{~h}$ \\
\hline
\end{tabular}

by Kasper et al. [19] and the experimental studies by Deponte et al. [8], the self-cleaning effect can be observed in the present study with locally resolved fouling resistances. Furthermore, the PFQ method provides insights into the dimple itself. The surface of the dimple has not yet been examined experimentally.

Figure 9 shows the local distribution of the fouling resistance matrix $R_{f, p f q}$ on the surface of the test plate inside and around the dimple. The area downstream of the dimple is less covered with particles than the area upstream of the dimple. The characteristic fouling pattern, observed in previous studies, is visible and quantifiable by the PFQ results. Figure 10 shows the results of the numerical investigation [19]. Viz, the trail downstream of the dimple is made visible with the numerical calculation, as well as with the PFQ method.

The local analysis of the fouling layer distribution is performed by extracting parts of the $\mathrm{R}_{\mathrm{f} \text {,pfq }}$ matrix. Figure 11 shows an elevated fouling resistance on the outer edge of the plate and a constant value on the central surface upstream of the dimple. This effect is described by the boundary condition of the flow. The decreasing flow velocity in the boundary layer near the wall increases the propensity of particle deposition. The reduction of the value at the far outer edge of the plate shows the small area in which the plate is clamped in the channel. The core flow has a constant velocity in a turbulent flow regime; thus, the fouling resistance of about $4.8 \times$ $10^{7} \mathrm{~m}^{2} \mathrm{~K} / \mathrm{W}$ is also constant in this area.

The PFQ method allows for local investigation of the fouling resistance inside the dimple. Figure 12 shows the cross section in the flow direction through the center, from the front to the end of the dimple. Due to the concave geometry of the

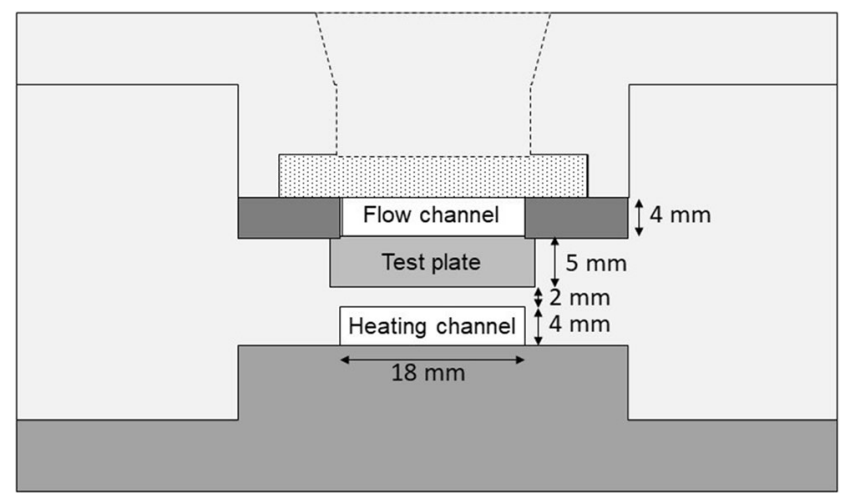

Fig. 6 Cross section of the flow and heating channel with the test plate in between 


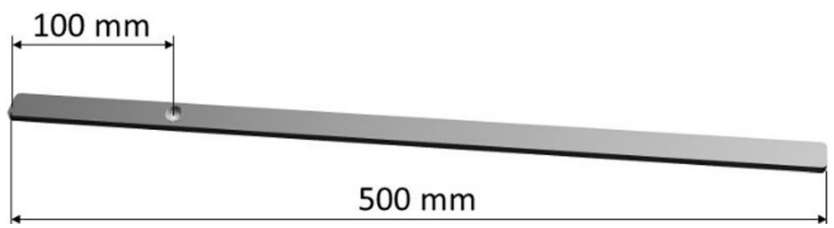

Fig. 7 Test plate with a single dimple

dimple, its surface is greater than the area shown on the obtained image. This surface enlargement is considered in the calculation of the fouling resistance. The highest fouling resistance is measured at the surface of the dimple and is comparable to the area upstream of the dimple. On the further surface of the dimple, the deposited mass is slightly reduced to an average fouling resistance of about $4.0 \times 10^{7} \mathrm{~m}^{2} \mathrm{~K} / \mathrm{W}$.

The area of a pixel projected onto the plate surface is $0.03 \mathrm{~mm} \times 0.03 \mathrm{~mm}$. The results show that the local differentiation of the distribution of fouling resistances is very accurate, so the self-cleaning effect of the dimples can be quantified. If necessary, the resolution of the PFQ method can easily be increased by using other lenses or a camera with a larger sensor.

\subsection{Comparison of methods}

The three applied methods are compared by measuring each fouling resistance after $60 \mathrm{~min}$ on the S26B test plate with one dimple. The mean value of five measurements for each method is shown in Fig. 13. The thermal fouling resistance is measured integrally over the whole plate. The other two methods are applied upstream of the dimple, so the influence of the dimple is not considered in those values.

All three methods provide comparable results, while the standard deviations are clearly different. The thin fouling layers just slightly decrease the overall heat transfer coefficient, and the change in the resulting temperature difference is low; therefore, the uncertainty in the thermal fouling resistance $\mathrm{R}_{\mathrm{f}, \mathrm{th}}$ is high. However, the mean value of the five

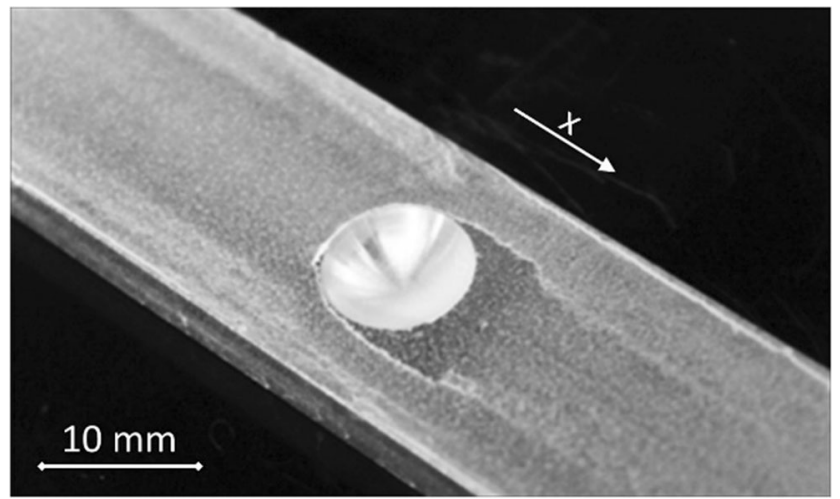

Fig. 8 Characteristic fouling pattern on a dimpled surface, with a visible trail downstream of the dimple; the fluid flow is in the $\mathrm{x}$ direction [19]

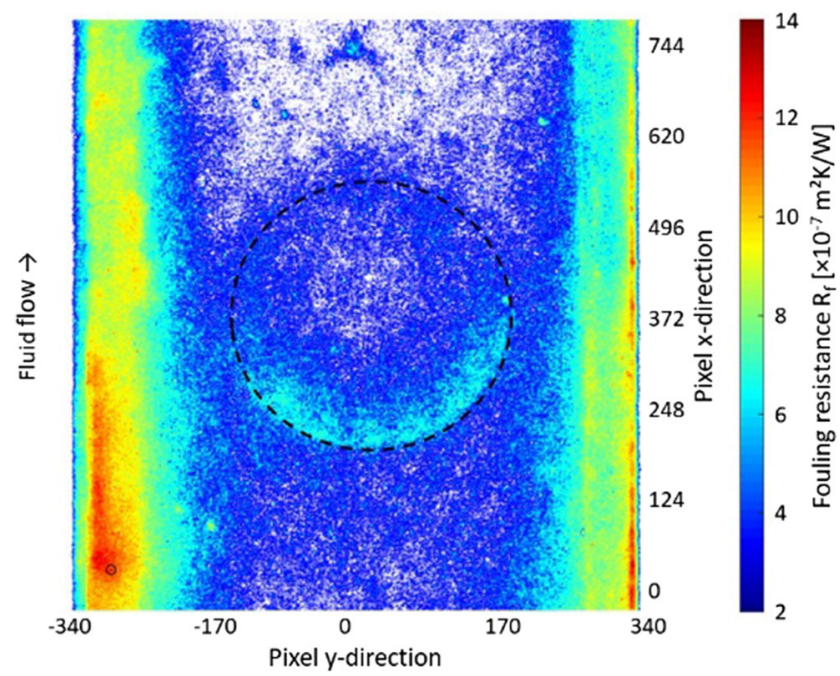

Fig. 9 Local fouling resistances around and inside the dimple with PFQ; fluid flow is in the $\mathrm{x}$ direction

measurements is comparable with the values from the other methods.

While the application of all three methods is straightforward, the analytical effort varies. The thermal fouling resistance can be calculated online with the direct output of the process control system. The PFQ method needs images captured during the process, and these images have to be evaluated offline. This is done with a MatLab ${ }^{\circledR}$ procedure. The mass-based method requires the most analytical effort after performing the experiment.

Next to the analytical effort, the instrumental effort has to be considered. Both the PFQ and the mass-based method do not need a heat transferring surface. The provided heating channel is only necessary for the determination of the thermal fouling resistance.

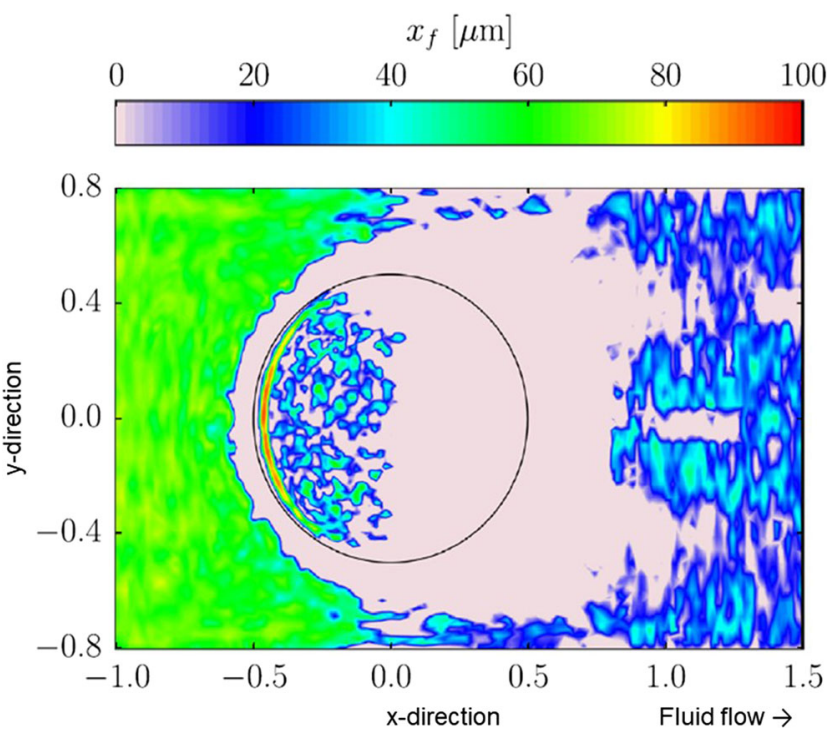

Fig. 10 Distribution and thickness $\mathrm{x}_{\mathrm{f}}$ of the fouling layer with CFD [19] 


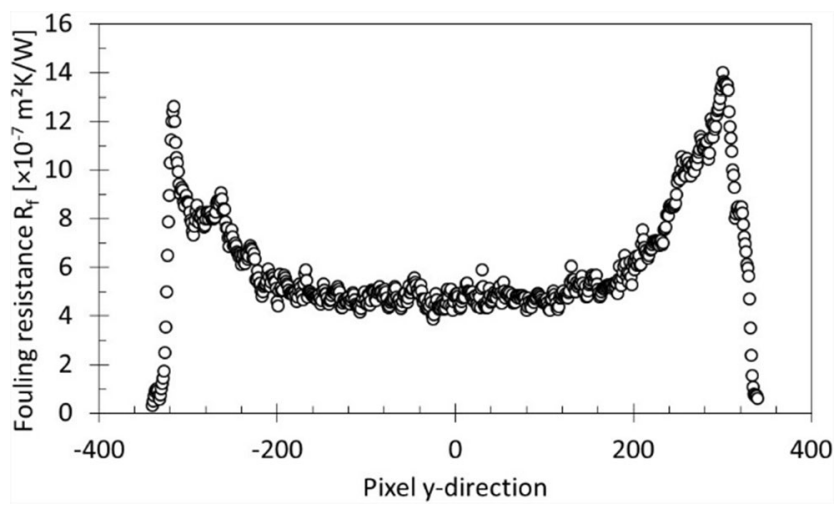

Fig. 11 Fouling resistance upstream of the dimple (mean values of pixels-lines no. 125 to 130 (Fig. 9) in x-position)

In contrast to the other methods, the PFQ has certain requirements for the fouling material. The particles must be able to provide a detectable phosphorescence. For fundamental studies of particulate fouling, phosphorescent particles of various sizes and densities are available. The investigation of other fouling mechanisms, like crystalline or bio fouling, also requires additional tracer particles if no phosphorescence is provided by the system itself. The PFQ also may not be suitable for very thick fouling layers. For thin layers of particles, the emitting light of the particles of lower layers can shine through and be detected. If several layers form on top of each other, the light emitted by the lower layers may not be detected by the camera. The threshold value of the layer thickness, from which the light can no longer be detected by the cameras, must be known for the used phosphorescent particles, and can be determined via calibration with defined layer thicknesses.

The application of the PFQ also needs a calibration for each fouling problem to calculate fouling resistances. Furthermore, the maximal detectable height of the layer and the light intensity must be known to ensure detection of the emitted light of all the layers.

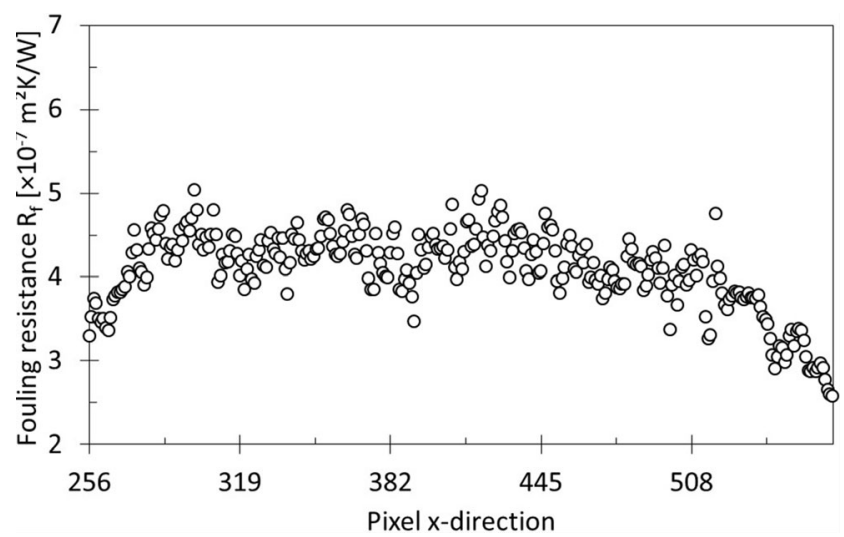

Fig. 12 Cross section in the flow direction, from the front to end through the center line of the dimple

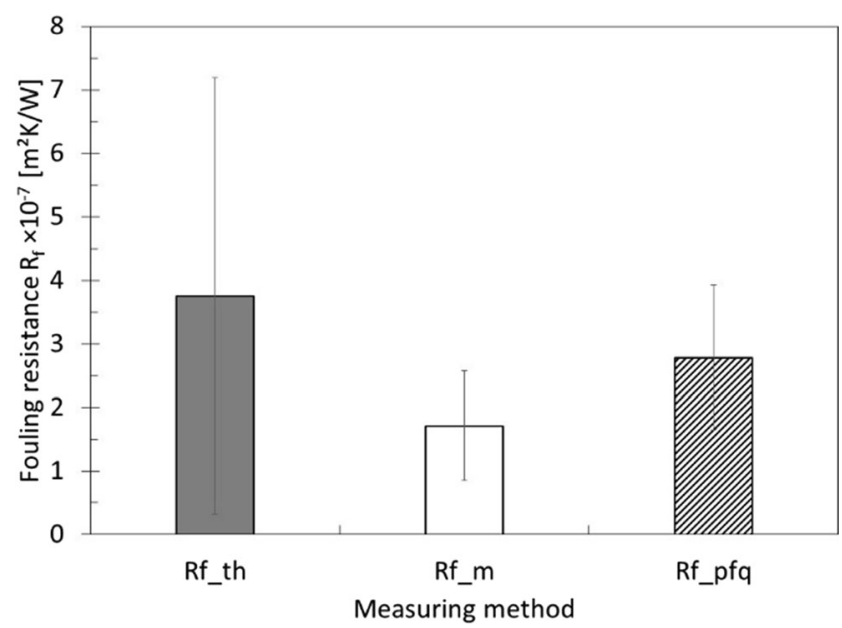

Fig. 13 Comparison of the measured fouling resistance with different methods

\subsection{Time-resolved fouling progression}

After the experiment, a fouling layer on each test plate is clearly visible. Observation through the glass windows in the lid of the channel suggests that the fouling layer grows at a constant rate. This assumption is supported by the course of the measured fouling resistance. The progression of the thermal fouling resistance and the fouling resistance measured with the PFQ are shown in Fig. 14 for the S26B test plate during a single fouling experiment. The PFQ fouling resistance is measured online through the windows of the lid of the channel and is the mean value for an area of $80 \mathrm{~mm} \times$ $18 \mathrm{~mm}$ on the test plate with the dimple in the middle of the image.

The evaluation of the two methods shows a comparable course for the increase in fouling resistance over time. On average, the thermal fouling resistance is slightly higher than $\mathrm{R}_{\mathrm{f}, \mathrm{pfq}}$ because of the area used for the analysis. The integral thermal fouling resistance refers to the total surface of the

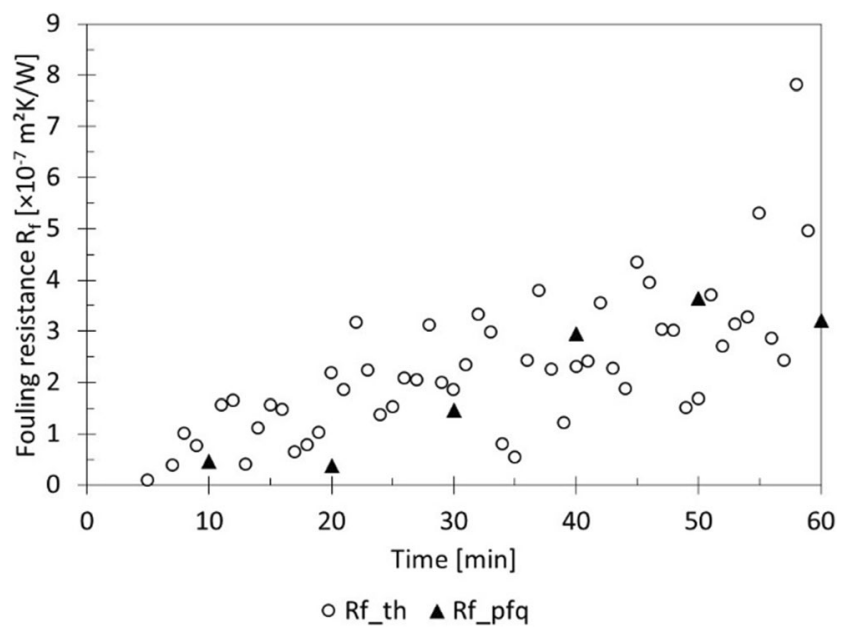

Fig. 14 Comparison of the progression of fouling resistance of a single experiment on S26B measured thermally and with PFQ 
plate, so the area influenced by the dimple is comparatively small. This is the area where the self-cleaning takes place, so the local quantification is described in the next section.

An asymptotic behavior of the fouling resistance is assumed but cannot be clearly verified in this experiment of one hour duration. The fouling resistance still seems to increase, although at a slower rate than at the beginning of the experiment. For a final evaluation, the measuring period must be increased.

\subsection{Locally resolved fouling resistance}

The determination of the mass-based fouling resistance is invasive, but it is the only direct measuring method for fouling deposits. It does not need a tracer or a heat transferring surface for the determination of the fouling resistance.

The particulate deposition can be resolved locally with the mass-based fouling resistance, as well as with the fouling PFQ method (e.g., upstream, and downstream of the dimple) to confirm the statements of previous studies that claim a selfcleaning of dimpled surfaces $[8,19]$. The results of the massbased fouling resistance and the fouling resistance measured with the PFQ upstream and downstream of the dimple are shown in Fig. 15.

The expected lower fouling resistance downstream of the dimple due to the self-cleaning effect can be measured with both methods. With reference to the fouling resistance upstream of the dimple, the self-cleaning with respect to the reduction of the fouling resistance is $33.3 \%$ for the massbased method and $46.9 \%$ for the PFQ method. The underestimation of the mass-based fouling resistance can be explained by an incomplete extraction of the fouling layer with the adhesive tape. This is especially the case for the measurement of thick layers.

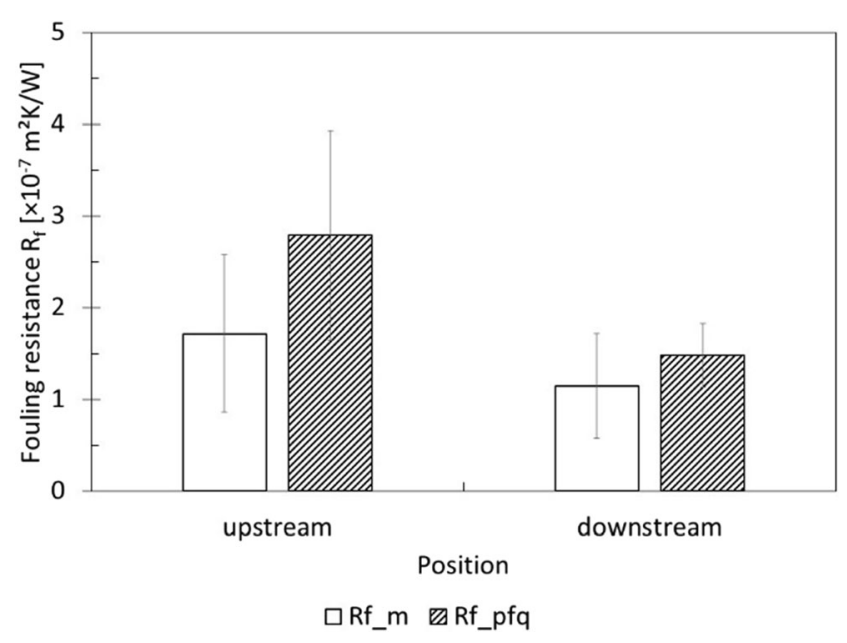

Fig. 15 Comparison of the mass-based fouling resistance and the fouling resistance measured with PFQ up- and downstream of the dimple
The higher precision of the PFQ method is due to the measuring technique: While the observed area of the mass-based method is limited to the minimum measurable and weighable size of the adhesive tape, the PFQ allows even smaller local distinctions and is limited only by the resolution of the camera sensor. Furthermore, the application of adhesive tape on structured surfaces may not be possible due to shape and size of the structuring. This limitation does not apply to the PFQ method. Even in cavities that are difficult to access, the measurement of fouling resistances is possible if optical accessibility is available.

\section{Conclusions}

The PFQ method is shown to be well applicable for the evaluation of the local fouling behavior of structured surfaces such as dimples. Even though the thermal, mass-based, and PFQ methods are all applicable for the measurement of the fouling resistance, the PFQ has some unique advantages, including the ability to carry out measurements online with a high local resolution. However, its applicability inherently depends on the light emitted from the fouling layer. Another advantage is that the PFQ does not need a heat transferring surface to measure the fouling resistances, unlike the thermally measured fouling resistance. Moreover, the PFQ method is not invasive, like the mass-based fouling resistance method, so the fouling process is not interrupted while the results are collected online. A further advantage is that the fouling resistances can be calculated automatically during the experiment.

The PFQ method can be evaluated as the most accurate method based on the measurements performed here. Therefore, it is recommended for the high-resolution evaluation of structured surfaces.

The suitability of the PFQ method for fundamental research was shown in this study. Further investigations with different dimple geometry and multiple dimples in a row on the test plate will be conducted with this method. The method will also be validated with more results from numerical simulations of particle deposition on dimpled surfaces.

Acknowledgements The authors thank the German Research Foundation (Deutsche Forschungsgemeinschaft DFG, grant no. SCHO 842/19-2) for financial support.

Funding Open Access funding enabled ad organized by Projekt DEAL.

\section{Declarations}

Conflict of interest On behalf of all authors, the corresponding author states that there are no conflicts of interest. 
Open Access This article is licensed under a Creative Commons Attribution 4.0 International License, which permits use, sharing, adaptation, distribution and reproduction in any medium or format, as long as you give appropriate credit to the original author(s) and the source, provide a link to the Creative Commons licence, and indicate if changes were made. The images or other third party material in this article are included in the article's Creative Commons licence, unless indicated otherwise in a credit line to the material. If material is not included in the article's Creative Commons licence and your intended use is not permitted by statutory regulation or exceeds the permitted use, you will need to obtain permission directly from the copyright holder. To view a copy of this licence, visit http://creativecommons.org/licenses/by/4.0/.

\section{References}

1. Müller-Steinhagen H (2011) Heat transfer fouling: 50 years after the Kern and Seaton model. Heat Transfer Eng 32:1-13. https://doi. org/10.1080/01457632.2010.505127

2. Bott T (1995) Fouling of heat exchangers. Elsevier Science and Technology Books, Birmingham. https://doi.org/10.1016/B978-0444-82186-7.X5000-3

3. Krause S (1993) fouling of heat transfer surfaces by crystallization and sedimentation. Int Chem Eng 33:335-401

4. Grandgeorge S, Jallut C, Thonon B (1998) Particulate fouling of corrugated plate heat exchangers. Global kinetic and equilibrium studies. Chem Eng Sci 53:3051-3071. https://doi.org/10.1016/ S0009-2509(98)00128-6

5. Oliveira R (1997) Understanding adhesion: A means for preventing fouling. Exp Therm Fluid Sci 14:316-332. https://doi.org/10.1016/ S0894-1777(96)00134-3

6. Kern D, Seaton R (1959) A theoretical analysis of thermal surface fouling. Br Chem Eng 14:258-262

7. Müller-Steinhagen H, Reif F, Epstein N, Watkinson A (1988) Influence of operating conditions on particulate fouling. Can $\mathrm{J}$ Chem Eng 66:42-50. https://doi.org/10.1002/cjce.5450660106

8. Deponte H, Rohwer L, Augustin W, Scholl S (2019) Investigation of deposition and self-cleaning mechanism during particulate fouling on dimpled surfaces. Heat Mass Transfer 55:3633-3644. https://doi.org/10.1007/s00231-019-02676-0

9. Bohnet M (1987) Fouling of heat transfer surfaces. Chem Eng Technol 10:113-125. https://doi.org/10.1002/ceat.270100115

10. Brahim F (2003) Numerische Simulation des Kristallwachstums auf wärmeübertragenden Flächen (Fouling). Shaker Verlag, Aachen
11. Smart E, Moore L (2005) Solid state chemistry: An introduction, 3rd edn. Taylor \& Francis, Boca Raton

12. Krischer O (1956) Die wissenschaftlichen Grundlagen der Trocknungstechnik. Springer-Verlag, Berlin Heidelberg

13. Wu J, Zheng G, Lee LM (2012) Optical imaging techniques in microfluidics and their applications. Lab Chip 19:3566-3575. https://doi.org/10.1039/C2LC40517B

14. Köhler H, Stoye H, Mauermann M, Majschak J-P (2013) Optimization approach for efficient cleaning with impinging jets influence of nozzle diameter, pressure and nozzle distance. In: Proceeding of International Conference on Heat Exchanger Fouling and Cleaning, Budapest, Hungary, pp 421-428

15. Gerhards C, Schmidt A (2013) Assessing the cleanability of stainless steel surfaces - development of a testing method for starch and protein based soils. J Hyg Eng Des 3:9-14

16. Schöler M, Fuchs T, Helbig M, Augustin W, Scholl S, Majschak JP (2009) Monitoring of the local cleaning efficiency of pulsed flow cleaning procedures. Proc. 8th Int. Conf. on Heat Exchanger Fouling and Cleaning, Schladming, Austria, pp 455-463

17. Gordon P, Schöler M, Föste H, Helbig M, Augustin W, Chew J, Scholl S, Majschak J-P, Wilson DI (2014) A comparison of local phosphorescence detection and fluid dynamic gauging methods for studying the removal of cohesive fouling layers: Effect of layer roughness. Food Bioprod Process 92:46-53

18. Wagner W, Kretzschmar HJ, Span R, Krauss R (2010). VDI Heat Atlas - D2 Properties of selected important pure substances, VDI Heat Atlas. Springer-Verlag Berlin Heidelberg. https://doi.org/10. 1007/978-3-540-77877-6

19. Kasper R, Deponte H, Michel A, Turnow J, Augustin W, Scholl S, Kornev N (2018) Numerical investigation of the interaction between local flow structures and particulate fouling on structured heat transfer surfaces. Int J Heat Fluid Flow 71:68-79. https://doi. org/10.1016/j.ijheatfluidflow.2018.03.002

20. Kovalenko G, Terekhov V, Khalatov A (2010) Flow regime in a single dimple. J Appl Mech Tech Phys 51:839-848

21. Ebrahimi A, Naranjani B (2016) An investigation on thermohydraulic performance of a flat-plate channel with pyramidal protrusions. Appl Therm Eng 106:316-324. https://doi.org/10.1016/j. applthermaleng.2016.06.000

22. Turnow J, Kornev N, Isaev S, Hassel E (2011) Vortex mechanism of heat transfer enhancement in a channel with spherical and oval dimples. Heat Mass Transfer 47:301-313. https://doi.org/10.1007/ s00231-010-0720-5

Publisher's note Springer Nature remains neutral with regard to jurisdictional claims in published maps and institutional affiliations. 